\title{
Optimal risk management in defined benefit stochastic pension funds ${ }^{\text {th }}$
}

\author{
Ricardo Josa-Fombellida a , Juan Pablo Rincón-Zapatero ${ }^{\text {b }}$ \\ a Dpto. de Estadística e Investigación Operativa, Universidad de Valladolid, Avda. Valle Esgueva 6, 47011 Valladolid, Spain

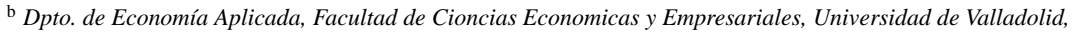 \\ Avda. Valle Esgueva 6, 47011 Valladolid, Spain
}

\begin{abstract}
We consider a continuous time dynamic pension funding model in a defined benefit plan of an employment system. The benefits liabilities are random, given by a geometric Brownian process. Three different situations are studied regarding the investment decisions taken by the sponsoring employer: in the first, the fund is invested at a constant, risk-free rate of interest; in the second, the promoter invests in a portfolio with $n$ risky assets and a risk-free security; finally, it is supposed that the rate of return is stochastic. Modelling the preferences of the manager such that the main objective is to minimize both the contribution rate risk and the solvency risk, we study cases where the optimal behavior leads to a spread method of funding.
\end{abstract}

JEL classification: G23; G11

Subj. classification: E13; B81

Keywords: Defined benefit pension fund; Contribution rate risk; Solvency risk; Asset allocation; Stochastic control

\section{Introduction}

The dynamically optimal management of defined benefit pension plans has considerable economic interest, due to the great importance that the world of pensions provision has acquired in financial markets.

In this paper we analyze the optimal contribution rate and asset allocation decisions for a manager of a defined benefit pension plan who wishes to keep the fund as close as possible to prescribed targets. These targets are fixed by actuarial cost methods, allowing an ideal contribution rate — the normal cost—and an ideal fund level — the actuarial liability - to be defined. These ideal levels guarantee the benefits promised to members incorporated to the pension plan over time. However, the existence of uncertainty in some elements of the plan or in the rate of return of the

\footnotetext{
We are grateful to the anonymous referee who carefully read the manuscript and suggested a number of improvements

* Corresponding author Tel : +34-983-186-591; fax: +34-983-423-299

E-mail address: zapatero@eco uva es (J P Rincón-Zapatero)
} 
assets of the fund can cause the evolution of the plan to be quite different from the initially designed valuation. Hence the contribution rate must be the normal cost modified by a suitable supplementary cost reflecting the disturbances. The supplementary cost is chosen by the promoter with the aim of bringing the expected value of the unfunded actuarial liability to zero. The instruments used by the manager to reach the objectives are the contribution rate and investment earnings.

Along the lines of Haberman (1993), Haberman and Sung (1994), Haberman (1997), Josa-Fombellida and Rincón-Zapatero (2001), we suppose that the aim of the controller is to minimize a combination of the contribution rate risk and the solvency risk. The former represents the size of deviation of contributions from the normal cost and is related with the stability of the plan. The latter risk is an indicator of the plan's safety, measuring deviations of the fund from the actuarial liability. The model is considered on an unbounded horizon and with a positive discount rate, meaning that the sponsor is more worried about the short-term than with the long run behavior of the fund.

Josa-Fombellida and Rincón-Zapatero (2001) considered the case of a constant value for the benefits, in a context closely related with the framework pioneered by Merton (1971) for optimal consumption and portfolio selection. That paper found that the optimal behavior of the controller leads to a spread method of funding if the technical rate of actualization of the actuarial liability equals the risk-free rate of return. With a spread method the supplementary cost is proportional to the unfunded actuarial liability, in such a way that the corrections made in the rate of contribution to the normal cost are small when the fund is close to the target. Furthermore, it enjoys good stability properties. This is why spread methods have became popular with professionals and institutional agents.

Haberman and Sung (1994) considered a similar model in discrete time, on a finite horizon both in deterministic and stochastic frameworks. These authors do not contemplate investment as an instrumental variable, but all the fund assets are invested at a random rate of return. Another difference with our paper is that they consider constant benefits, whereas in our case we allow stochastic benefits.

Other related papers are O'Brien (1987), Boulier et al. (1995, 1996), Cairns (1995, 1996, 2000), Owadally and Haberman (1999), Vigna and Haberman (2001), Taylor (2002), Chang et al. (2003). Our paper extends the previous analysis incorporating a source of uncertainty in the benefit outgo, supposing that the benefits are given by a geometric Brownian motion. O'Brien (1987) analyzes a stochastic optimal control problem, where the uncertainty in the benefits is modelled in a quite different way. This author makes a linear approximation to the exponential fund model, see Bowers et al. (1986), to retain analytical tractability of the problem. However, no investment decisions are available for the manager, who wishes to maintain a constant fund ratio (with respect to the actuarial liability), and penalizes fluctuations of the contribution rate from zero.

Our paper contemplates three different situations which are studied regarding the investment decisions taken by the sponsoring employer: (i) in the first, the fund is invested at a constant rate of interest; (ii) the promoter invests in a portfolio with $n$ risky assets and a risk-free security; (iii) finally, it is supposed that the rate of return is stochastic. Note that the benefits are a non-tradable process, hence the market is incomplete and, furthermore, we also consider the existence of correlation between the sources of uncertainty in the benefits and in the asset returns. However, the consideration of the benefits as geometric Brownian motion is fundamental to our approach. The problem is solved under particular assumptions-depending on the scenario-concerning the technical rate of actualization and the evolution of the liabilities. These hypotheses are motivated by our intention to show that spread methods lead to a minimization of risk in pension funding, even in a stochastic environment.

An outline of the paper and a summary of the main results are given next. Section 2 provides the definitions of the main elements incorporated in a defined benefit pension plan of an employment system. The actuarial functions, necessary for the valuation of the plan are also introduced, and we prove that when the benefits are given by a geometric Brownian motion, the related actuarial functions are processes of the same type.

Section 3 considers that the fund is invested at a constant and deterministic rate of interest. We find that if the rate of return of the bond is chosen as the valuation rate, then the optimal management of the fund is a spread method. Section 4 presents a model with $n+1$ financial assets, where one of them is a bond. The controller can buy and sell the assets without limitations, with the possibility of selling short and borrowing at the riskless rate of interest. The 
source of uncertainty of the benefits is correlated with that of the prices of the risky assets. This fact means, that in order to find a spread method of funding, the rate of return of the bond plus a correction term due to the existence of correlation must be chosen as the valuation rate. It can also be seen that in case (ii) the fund stabilizes faster than in case (i), and with a lesser total contribution.

In Section 5, we suppose that the fund is totally invested in a single risky asset. This poses an interesting problem, because it is not clear how the technical rate of actualization could be chosen to obtain a spread method.

Section 6 is devoted to establishing some concluding remarks. All the proofs are in Appendix A.

\section{The pension model and preliminary results}

The pension model under consideration refers to an aggregate pension fund whose funding scheme is of defined benefit type. In a defined benefit plan the benefits are established in advance by the manager. Strictly speaking, this can only be done in a deterministic setting. If there is uncertainty in some of the elements of the plan, as for example, in its population, in the salary growth rate or in the appreciation of pension retirements, then the best the sponsor can do is to model the uncertainty. The contributions are instruments used to maintain the fund within adequate levels.

In this paper the actuarial valuation to estimate the main components of the plan are done at each instant of time. We denote $F(t)$ as the value of the assets of the fund at time $t ; C(t)$ is the contribution rate of the sponsor needed to accrue the amount of the defined benefits at the moment of retirement; the defined benefits are denoted by $P(t)$; the normal cost for all participants by $\mathrm{NC}(t)$; the actuarial liability by $\operatorname{AL}(t)$; the unfunded actuarial liability by $\operatorname{UAL}(t)=\mathrm{AL}(t)-F(t)$ and the supplementary contribution rate amortizing $\operatorname{UAL}(t)$ at time $t$, by $\mathrm{SC}(t)=C(t)-\mathrm{NC}(t)$. The valuation of the plan is done with a constant rate of interest $\delta$, called the technical rate of interest.

Josa-Fombellida and Rincón-Zapatero (2001) considered the values of $P$, NC and AL were constants. A more realistic assumption is to suppose the existence of disturbances that affect the evolution of the benefits and hence the evolution of the normal cost and the actuarial liability. To model this possibility, we consider a probability space $(\Omega, \mathcal{F}, \mathbb{P})$, where $\mathcal{F}=\left\{\mathcal{F}_{t}\right\}_{t \geq 0}$ is a complete and right continuous filtration generated by the one-dimensional Brownian motion $\{B(t)\}_{t \geq 0}$ and $\mathbb{P}$ is a probability measure on $\Omega$. One of the more general hypotheses about the behavior of $P$ is to suppose that $P$ is a path-continuous scalar Itô process defined on $(\Omega, \mathcal{F}, \mathbb{P})$ as

$$
P(t)=P_{0}+\int_{0}^{t} \mu(P(s), s) \mathrm{d} s+\int_{0}^{t} \eta(P(s), s) \mathrm{d} B(s), \quad t \geq 0,
$$

where $P_{0}$ represents the initial liabilities. This formulation allows us to consider that the population of the plan is subject to disturbances that cannot be completely anticipated by the sponsor.

Following Bowers et al. (1986), we introduce the hypothesis that the benefits accumulated by the worker throughout his or her life are distributed according to the distribution function $M$, with an associated density function $m$. For age $x$, the value $M(x)$ represents the percentage of the actuarial value of the future benefits accumulated until $x$. The support of $m$ is the fixed interval $[a, d]$, hence $m(x)=0$, if $x \leq a$ or $x \geq d$. We are supposing that all the participants enter the plan at age $a$, whereas the common age of retirement is $d$.

Along the lines of the deterministic case, the stochastic actuarial liability and the stochastic normal cost are defined as follows:

$$
\mathrm{AL}(t)=\int_{a}^{d} \mathrm{e}^{-\delta(d-x)} M(x) \mathbb{E}\left(P(t+d-x) \mid \mathcal{F}_{t}\right) \mathrm{d} x, \quad \mathrm{NC}(t)=\int_{a}^{d} \mathrm{e}^{-\delta(d-x)} m(x) \mathbb{E}\left(P(t+d-x) \mid \mathcal{F}_{t}\right) \mathrm{d} x
$$

for every $t \geq 0$, where $\mathbb{E}\left(\cdot \mid \mathcal{F}_{t}\right)$ denotes conditional expectation with respect to the filtration associated to the standard Brownian motion $\{B(t)\}_{t \geq 0}$. Thus, to compute the actuarial functions at time $t$, the manager makes use of 
the information available up to that time, in terms of the conditional expectation. The information in resumed in the corresponding element of the filtration, $\mathcal{F}_{t}$. Since $P$ is a diffusion process, it satisfies the Markov property (see Øksendal (1998)), hence conditional expectation with respect to the filtration equals conditional expectation with respect the current values of $P$ at time $t$. It is plausible to think that in the task of computing the ideal values of the fund, the information given by the evolution of the random source will be used.

For analytical tractability, we will need a more concrete specification for $P$. A typical way of modelling $P$ in the certain case is to postulate exponential growth, see Bowers et al. (1986). The stochastic counterpart is to consider the benefits outgo as a geometric Brownian motion. This is the content of the following hypothesis.

Hypothesis A. The benefits $P$ satisfies

$$
\mathrm{d} P(t)=\mu P(t) \mathrm{d} t+\eta P(t) \mathrm{d} B(t), \quad t \geq 0,
$$

where $\mu \in \mathbb{R}$ and $\eta \in \mathbb{R}_{+}$. The initial condition $P(0)=P_{0}$ is a random variable that represents the initial liabilities.

Hence we are supposing that the benefits increase or decrease on average at a constant exponential rate. The behavior of the actuarial functions $\mathrm{AL}$ and $\mathrm{NC}$ are then given in the following proposition.

Proposition 2.1. Under Hypothesis A there are constants $\psi_{\mathrm{AL}}$ and $\psi_{\mathrm{NC}}$ such that $\mathrm{AL}=\psi_{\mathrm{AL}} P$ and $\mathrm{NC}=\psi_{\mathrm{NC}} P$. Furthermore, $\psi_{\mathrm{NC}}=1+(\mu-\delta) \psi_{\mathrm{AL}}$ and the identity $(\delta-\mu) \mathrm{AL}(t)+\mathrm{NC}(t)-P(t)=0$ holds for every $t \geq 0$.

From the proposition we deduce that

$$
\mathrm{dAL}(t)=\mu \mathrm{AL}(t) \mathrm{d} t+\eta \operatorname{AL}(t) \mathrm{d} B(t), \quad \operatorname{AL}(0)=\psi_{\mathrm{AL}} P_{0},
$$

and also $\mathrm{dAL}(t)=(\delta \mathrm{AL}(t)+\mathrm{NC}(t)-P(t)) \mathrm{d} t+\eta \operatorname{AL}(t) \mathrm{d} B(t)$. This is an analogous equation to that appearing in the deterministic case, see Bowers et al. (1986).

\section{Optimal funding with safe investment}

A first approximation to the management of a defined benefit pension plan is to consider that the whole wealth of the fund is invested at a safe and constant rate of return. This case is studied here, where the unique source of uncertainty comes from the benefits outgo. Although the results could be obtained from the next section, we analyze this case separately to isolate more clearly the effects of investment decisions and to facilitate comparison.

We consider a bond $S^{0}$ verifying

$$
\mathrm{d} S^{0}(t)=r S^{0}(t) \mathrm{d} t, \quad S^{0}(0)=1,
$$

where $r>0$ is the constant rate of interest.

The evolution of the fund is given by the ordinary differential equation

$$
\mathrm{d} F(t)=(r F(t)+C(t)-P(t)) \mathrm{d} t, \quad t \geq 0
$$

with initial condition $F(0)=F_{0}>0$.

In the control problem considered here, the only instrument of the manager is the rate of contribution $C$, that we suppose is a measurable adapted process with respect to $\left\{\mathcal{F}_{t}\right\}$ satisfying

$$
\int_{0}^{\infty}|C(s)| \mathrm{d} s<\infty \quad \text { a.s. }
$$


We will use Proposition 2.1 and the definition of the supplementary cost to eliminate the processes $P$ and NC in the control problem. The equality $(\delta-\mu) \mathrm{AL}+\mathrm{NC}-P=0$ allows us to write (2) as

$$
\mathrm{d} F(t)=(r F(t)+\mathrm{SC}(t)+\mathrm{NC}(t)-P(t)) \mathrm{d} t=(r F(t)+\mathrm{SC}(t)+(\mu-\delta) \operatorname{AL}(t)) \mathrm{d} t, \quad t \geq 0 .
$$

Let us now turn to the preferences of the controller. We assume that he or she wishes to minimize a convex combination of the contribution rate risk and the solvency risk. Thus, the objective functional to be minimized over the class of admissible controls $\mathcal{A}_{F_{0}, \mathrm{AL}_{0}}$, is given by

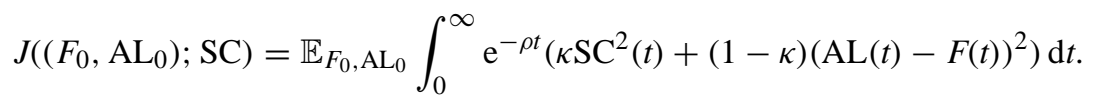

Note that we choose $\mathrm{SC}$ as the control variable instead of $C$, leading to an equivalent control problem. Here, $\mathcal{A}_{F_{0}}, \mathrm{AL}_{0}$ is the set of measurable processes $\mathrm{SC}=C-\mathrm{NC}$, where $C$ satisfies (3), and $F$ and AL satisfy (4) and (1), respectively. In the above, $\mathbb{E}_{F_{0}, \mathrm{AL}_{0}}$ denotes conditional expectation with respect to the initial conditions $\left(F_{0}, \mathrm{AL}_{0}\right)$.

The parameter $\kappa, 0<\kappa \leq 1$, is a weighting factor reflecting the relative importance for the employer of the two different types of risks. Note that the specification (5) implies that the fund manager assigns the same importance to over and under deviations of the fund's assets and contributions from their respective targets. The recent paper by Chang et al. (2003) considers the square of ratio deviation of the variables, plus linear terms in the performance criterion function, in such a way that under-funding and over-contributing is more penalized than over-funding and under-contributing.

An important issue is how to choose the technical rate of actualization $\delta$. Given that there is a riskless rate of interest $r$ in the market, a rather natural selection is $\delta=r$. This is better understood if a hypothetical situation is considered where the sponsor borrows money to satisfy his or her liabilities. The rate of borrowing is $r$, so the "correct" valuation of the debt is $\delta=r$. Hence we impose the following hypothesis.

Hypothesis B. The technical rate of actualization equals the riskless rate of interest, $\delta=r$.

It turns out that, in this case, the structure of the problem becomes quite simple and the optimal solution involves a spread method of funding. Of course, the problem could be easily solved for a different value of $\delta$, but in this case the optimal contribution rate is not proportional to the unfunded actuarial liability, UAL $=\mathrm{AL}-F$.

The dynamic programming approach is used to solve the problem. To make the process work, some properties of the value function need to be established. In particular, we prove that it is a solution to the Hamilton-Jacobi-Bellman equation (HJB henceforth). The value function is defined as

$$
\hat{V}(F, \mathrm{AL})=\min _{\mathrm{SC} \in \mathcal{A}_{F, \mathrm{AL}}}\{J((F, \mathrm{AL}) ; \mathrm{SC}): \text { s.t. }(4),(1)\} .
$$

Since the problem is autonomous and the horizon unbounded, we may suppose that $\hat{V}$ is time independent. It is clear that the value function so defined is non-negative and strictly convex.

The connection between value functions in optimal control theory (deterministic or stochastic) and optimal feedback controls is accomplished by the HJB equation, see Fleming and Soner (1993). We thus have the following result.

Theorem 3.1. Suppose that Hypotheses A and B hold. If the inequality

$$
2 \mu+\eta^{2}<\rho
$$

is satisfied, then the optimal contribution rate is given by

$$
C^{*}=\mathrm{NC}+\frac{\alpha_{F F}}{\kappa} \mathrm{UAL}
$$


where $\alpha_{F F}$ is the unique positive solution to the equation

$$
\alpha_{F F}^{2}+\kappa(\rho-2 r) \alpha_{F F}-\kappa(1-\kappa)=0 .
$$

Remark 3.1. From (7) in Theorem 3.1, the optimal supplementary cost is proportional to the unfunded actuarial liability: $\mathrm{SC}^{*}=\left(\alpha_{F F} / \kappa\right) \mathrm{UAL}^{*}$. The form of the solution suggests that the optimal behavior of the manager in a defined benefit pension plan, when he or she wishes to minimize the solvency and contribution risks, corresponds to what is called in the literature the spread method of funding. This fact was first pointed out by Haberman and Sung (1994) in a discrete time environment and corroborated by Josa-Fombellida and Rincón-Zapatero (2001) in continuous time. The spread method appears because of Hypothesis B.

Remark 3.2. From (A.2) in Appendix A and Arnold (1974, p. 139), we obtain

$$
\mathbb{E}_{F_{0}, \mathrm{AL}_{0}} F^{*}(t)-\mathbb{E}_{F_{0}, \mathrm{AL}_{0}} \mathrm{AL}(t)=\left(F_{0}-\mathrm{AL}_{0}\right) \mathrm{e}^{\left(r-\alpha_{F F} / \kappa\right) t} .
$$

Therefore, if the inequality

$$
\alpha_{F F}>\kappa r
$$

holds, then the difference between the expected value of the fund and the expected value of the actuarial liability converges to zero and from (7), the difference between the expected value of the contribution rate and the expected value of the normal cost tends to zero.

The constant $\alpha_{F F} / \kappa$ depends on $\kappa, r$ and $\rho$. In fact, if $r \geq \rho$ condition (9) holds. When $r<\rho$, (9) implies that

$$
\kappa<\frac{1}{1+r(\rho-r)},
$$

where we have used (8). This inequality places a constraint between the impatience rate of the controller-whenever it is greater than the rate of return of the bond-and the weight given to the objectives. It is clear that if $\rho$ increases, that is to say, the promoter is more concerned with the near future than with the long term, then the weight of the solvency risk term has to diminish to stabilize the plan. The contribution rate thus increases with $\rho$.

\section{Optimal funding with portfolio selection}

In this section the management of the pension fund is modelled in the environment established in the above sections, but the possibility for the manager to invest in risky assets is introduced. The sponsoring employer manages the funding process by making a portfolio choice of $n$ risky assets $S^{1}(t), \ldots, S^{n}(t)$ and a bond $S^{0}(t), 0 \leq t<\infty$, with dynamics given by the equations

$$
\begin{aligned}
& \mathrm{d} S^{0}(t)=r S^{0}(t) \mathrm{d} t, \quad S^{0}(0)=1, \\
& \mathrm{~d} S^{i}(t)=S^{i}(t)\left(b_{i} \mathrm{~d} t+\sum_{j=1}^{n} \sigma_{i j} \mathrm{~d} W_{j}(t)\right), \quad S^{i}(0)=s_{i}, \quad 1 \leq i \leq n .
\end{aligned}
$$

Here $b_{i}$ and $\sigma_{i j}, 1 \leq i, j \leq n$ are positive constants. The vector $\left(W_{0}(t), W_{1}(t), \ldots, W_{n}(t)\right)^{\top}$ is an $(n+1)$-dimensional standard Brownian motion defined on the probability space $(\Omega, \mathcal{G}, \mathbb{P})$, where $\left\{\mathcal{G}_{t}\right\}$ denotes the completion of the filtration $\sigma\left\{\left(W_{0}(s), W_{1}(s), \ldots, W_{n}(s)\right)^{\top}: 0 \leq s \leq t\right\}$.

We assume that $b_{i}>r$, for each $i=1, \ldots, n$. Given that the benefits $P$ are conditioned for the increase in salary of the sponsoring employees, we suppose the existence of correlation $q_{i} \in[-1,1]$ between the Brownians $B$ and $W_{i}$, for $i=1, \ldots, n$, which can be explained by the effects of salary on inflation and the effects of the latter on the asset prices. This means that $\mathbb{E}\left(B(t) W_{i}(s)\right)=q_{i} \min (t, s)$ for $i=1, \ldots, n$ and $B(t)=\sqrt{1-\bar{q}^{\top} \bar{q}} W_{0}(t)+\bar{q}^{\top} \bar{W}(t)$, 
where $\bar{W}(t)=\left(W_{1}(t), W_{2}(t), \ldots, W_{n}(t)\right)^{\top}$ and $\bar{q}=\left(q_{1}, q_{2}, \ldots, q_{n}\right)^{\top}$. When $\bar{q}^{\top} \bar{q} \neq 1$ the risk in the benefits outgo cannot be eliminated by trading in the financial market.

The matrix $\left(\sigma_{i j}\right)$ is denoted by $\sigma$ and the market price of risk, $\sigma^{-1}(\bar{b}-r \overline{1})$, by $\bar{\theta}$, where $\bar{b}=\left(b_{1}, \ldots, b_{n}\right)^{\top}$ and $\overline{1}$ is a (column) vector of 1's. We will suppose that the symmetric matrix $\Sigma=\sigma \sigma^{\top}$ is positive definite. $\lambda_{i}(t)$ denotes the quantity of the fund invested by the promoter in the asset $i$, for $0 \leq i \leq n$. The quantity $F-\sum_{i=1}^{n} \lambda_{i}$ is invested in the risk-free bond. There are no bounds on these variables. A negative value of $\lambda_{i}$ means that the promoter is selling short the corresponding stock. If $\sum_{i=1}^{n} \lambda_{i}$ is greater than the value of the fund, then the manager is borrowing money at rate $r$ to invest in the stocks. $\bar{\lambda}(t)$ denotes $\left(\lambda_{1}(t), \ldots, \lambda_{n}(t)\right)^{\top}$.

A portfolio process or trading strategy $\bar{\lambda}(t)$ is an $\mathbb{R}^{n}$-measurable process adapted to $\left\{\mathcal{G}_{t}\right\}$ such that

$$
\int_{0}^{\infty} \bar{\lambda}^{\top}(s) \bar{\lambda}(s) \mathrm{d} s<\infty \quad \text { a.s. }
$$

We suppose that changes in the fund level derive solely from changes in the asset prices, the interest of the bond, the contribution rate and the benefits. Consequently:

$$
\mathrm{d} F(t)=\sum_{i=1}^{n} \lambda_{i}(t) \frac{\mathrm{d} S^{i}(t)}{S^{i}(t)}+\left(F(t)-\sum_{i=1}^{n} \lambda_{i}(t)\right) \frac{\mathrm{d} S^{0}(t)}{S^{0}(t)}+(C(t)-P(t)) \mathrm{d} t .
$$

By (11), (12) and (14) we obtain that the fund amount satisfies the following stochastic differential equation:

$$
\mathrm{d} F(t)=\left(r F(t)+\sum_{i=1}^{n} \lambda_{i}(t)\left(b_{i}-r\right)+C(t)-P(t)\right) \mathrm{d} t+\sum_{i=1}^{n} \sum_{j=1}^{n} \lambda_{i}(t) \sigma_{i j} \mathrm{~d} W_{j}(t)
$$

with initial condition $F(0)=F_{0}$. Using Proposition 2.1, this can be rewritten as

$$
\mathrm{d} F(t)=\left(r F(t)+\sum_{i=1}^{n} \lambda_{i}(t)\left(b_{i}-r\right)+\mathrm{SC}(t)+(\mu-\delta) \mathrm{AL}(t)\right) \mathrm{d} t+\sum_{i=1}^{n} \sum_{j=1}^{n} \lambda_{i}(t) \sigma_{i j} \mathrm{~d} W_{j}(t) .
$$

It is important to remark that the model describes an incomplete market, because the stochastic benefits $P$ cannot be traded in the security market and therefore the manager cannot hedge the inherent risk.

We shall now proceed to establish the problem of the funding process management when the possibility of diversification in the investment is considered. As in Section 3, the problem is stated in terms of the fund wealth and the actuarial liability as the state variables. Naturally, we now add new control variables, given by the vector of investment decisions, to the supplementary cost.

The elements in the class of admissible controls, $\mathcal{A}_{F_{0}}, \mathrm{AL}_{0}$, are Markovian stationary pairs $(\mathrm{SC}, \bar{\lambda})$ adapted to the filter $\left\{\mathcal{G}_{t}\right\}_{t \geq 0}$, satisfying (3) and (13), such that (1) and (15) admit a unique solution, which is $\mathcal{G}_{t}$-measurable with continuous paths.

To solve the problem stated above the same steps are followed as in the section above. The value function is defined as

$$
\hat{V}(F, \mathrm{AL})=\min _{(\mathrm{SC}, \bar{\lambda}) \in \mathcal{A}_{F, \mathrm{AL}}}\{J((F, \mathrm{AL}) ;(\mathrm{SC}, \bar{\lambda})): \text { s.t. }(15),(1)\} .
$$

Our aim is to prove that even in this framework, the solution of the problem is again, as in Section 3, a spread method of funding. For this to be the case, the following assumption needs to be imposed on the actualization rate.

Hypothesis $\mathbf{B}^{\prime}$. The technical rate of actualization satisfies $\delta=r+\eta \bar{q}^{\top} \bar{\theta}$.

In contradistinction to Hypothesis $\mathrm{B}$, the risk-free rate $r$ is now modified to get rid of the sources of uncertainty due to the investment in risky assets that, we suppose, are correlated with the Brownian motion driving the benefits 
process. In fact, if there is no correlation, or if $\eta=0$, then $\delta=r$. Here are some arguments justifying the hypothesis, using asset valuation techniques, which are based on the certainty equivalent growth rate approach. We mainly follow Constantinides (1978) and Shimko (1992, pp. 43-47). To simplify, consider only one worker of age $x=x_{0} \in[a, d]$. The promise to pay $P(t)$ to the worker at the time of retirement $x=d$ is valued by the sponsor by

$$
\mathrm{e}^{-\delta\left(d-x_{0}\right)} \mathbb{E}\left(P\left(t+d-x_{0}\right) \mid \mathcal{F}_{t}\right)=\mathrm{e}^{(\mu-\delta)\left(d-x_{0}\right)} P(t),
$$

as proven in Proposition 2.1. Now, for this valuation to be "correct", that is to say, to be a risk-neutral valuation of the liability, it must be the case that

$$
\mu-\delta=-\left(r-\alpha^{*}\right),
$$

where $\alpha^{*}=\mu-\eta \bar{q}^{\top} \bar{\theta}$ is known as the certainty equivalent growth rate of $P . \alpha^{*}$ allows the growth rate of the underlying process $P$ to be adjusted and the expected value in a risk-neutral market to be calculated. From (16) we obtain our Hypothesis $\mathrm{B}^{\prime}$, that is, $\delta=r+\eta \bar{q}^{\top} \bar{\theta}$.

Theorem 4.1. Suppose that Hypotheses $\mathrm{A}$ and $\mathrm{B}^{\prime}$ hold. If the inequality

$$
2 \mu+\eta^{2}<\rho
$$

is satisfied, then the optimal rate of contribution and optimal investment in the risky assets are given by

$$
\begin{aligned}
& C^{*}=\mathrm{NC}+\frac{\beta_{F F}}{\kappa} \mathrm{UAL}, \\
& \bar{\lambda}^{*}=\Sigma^{-1}(\bar{b}-r \overline{1}) \mathrm{UAL}+\eta \sigma^{-\top} \bar{q} \mathrm{AL},
\end{aligned}
$$

respectively, where $\beta_{F F}$ is the unique positive solution to the equation

$$
\beta_{F F}^{2}+\kappa\left(\rho-2 r+\bar{\theta}^{\top} \bar{\theta}\right) \beta_{F F}-\kappa(1-\kappa)=0 .
$$

Remark 4.1. Note that (18) implies, as in Section 3, that the supplementary cost is proportional to the unfunded actuarial liability. However, the constant of proportionality is now higher than in the case where the sponsor invests only in the bond. This can be checked computing the solutions of the respective equations defining $\alpha_{F F}$ and $\beta_{F F}$. The explanation becomes from the existence of uncertainty in the financial assets. The disturbances in the fund due to the evolution of the prices of the risky assets makes it more difficult to keep the solvency risk at zero, causing a more severe adjustment in the contribution rate.

With reference to the optimal investment decisions, (19), we can distinguish two terms. The first is again proportional to UAL, but the second is a correction term, depending on the risk parameters of the model and on AL. The constant of proportionality in the first term is the so called optimal-growth portfolio strategy.

An interesting feature of the model is that the manager takes a greater risk when the wealth of the fund is far below the actuarial liability than when it is closer. This behavior, which seems to be counterintuitive, was analyzed by Cairns (2000). However, there is a rational explanation. The sponsor is concerned with the stabilization of the funding process around the targets, because of their aim to reduce the risks inherent to the funding process. Given that the mean returns of the risky assets are greater than the rate of return of the bond, the sponsor is willing to take a greater risk to guide the fund towards the target. Please also note that, even in the case where the fund exactly matches AL at some time, the investment in the risky assets is not null, as is the case when there is no uncertainty in the benefits, see Josa-Fombellida and Rincón-Zapatero (2001), or if there is no correlation.

Remark 4.2. From (A.5) in Appendix A and Arnold (1974, p. 139), we have

$$
\mathbb{E}_{F_{0}, \mathrm{AL}_{0}} F^{*}(t)-\mathbb{E}_{F_{0}, \mathrm{AL}_{0}} \mathrm{AL}(t)=\left(F_{0}-\mathrm{AL}_{0}\right) \mathrm{e}^{\left(r-\bar{\theta}^{\top} \bar{\theta}-\beta_{F F} / \kappa\right) t} .
$$


Therefore, if the inequality

$$
\beta_{F F}>\kappa\left(r-\bar{\theta}^{\top} \bar{\theta}\right)
$$

holds, then the difference between the expected value of the optimal fund and the expected actuarial liability converges in the long term to zero. A similar comment applies to the contribution and the normal cost.

Inequality (21) is automatically fulfilled if $r \geq \rho+\bar{\theta}^{\top} \bar{\theta}$. However, when $r<\rho+\bar{\theta}^{\top} \bar{\theta}$, it reduces to

$$
\kappa<\frac{1}{1+r\left(\rho+\bar{\theta}^{\top} \bar{\theta}-r\right)} .
$$

As can be seen, this is more restrictive than when the manager of the fund only invests in fixed rent, because $\bar{\theta}^{\top} \bar{\theta}>0$. Compare this with expression (10).

Remark 4.3. In the optimal solution (19), the manager borrows money at rate $r$ to invest in risky asset $i$ - that is to say, $\lambda_{i}>F^{*}$-when the level of the fund is below $k_{i} \mathrm{AL}$, where the constant $k_{i}$ is defined as

$$
k_{i}=\frac{\bar{e}_{i} \Sigma^{-1}(\bar{b}-r \overline{1})+\eta \bar{e}_{i} \sigma^{-\top} \bar{q}}{1+\bar{e}_{i} \Sigma^{-1}(\bar{b}-r \overline{1})}, \quad \bar{e}_{i}=(0, \ldots, \stackrel{i}{1}, 0, \ldots, 0)
$$

for all $i=1,2, \ldots, n$. The manager takes a short position in asset $i$ - that is to say, $\lambda_{i}^{*}<0-$ when the fund is above the value $k_{i}^{\prime} \mathrm{AL}$, where

$$
k_{i}^{\prime}=\frac{\bar{e}_{i} \Sigma^{-1}(\bar{b}-r \overline{1})+\eta \bar{e}_{i} \sigma^{-\top} \bar{q}}{\bar{e}_{i} \Sigma^{-1}(\bar{b}-r \overline{1})}>k_{i} .
$$

We can summarize the above in the following behavior for the optimal investment

$$
\bar{\lambda}^{*} \leq \overline{0} \Leftrightarrow F^{*} \geq k_{i}^{\prime} \mathrm{AL}, \quad \lambda_{i}^{*} \geq F \Leftrightarrow 0<F^{*} \leq k_{i} \mathrm{AL}, \quad 0<\lambda_{i}^{*}<F \Leftrightarrow k_{i} \mathrm{AL}<F^{*}<k_{i}^{\prime} \mathrm{AL} .
$$

That is, when the fund is over $k_{i}^{\prime} \mathrm{AL}$, the manager is selling short in asset $i$. When the fund is below $k_{i} \mathrm{AL}$, the manager is borrowing money at rate $r$. Observe that even in the case of over-funding, $F^{*}>\mathrm{AL}$, the optimal decision may be to borrow money to invest in stocks. For this to be the case, it is necessary the existence of positive correlation. To simplify matters, assume that there is only one stock and that $q \eta / \sigma>1$. Then $k_{1}>1$ and the inequality $\mathrm{AL}<F^{*}<k_{1} \mathrm{AL}$ could be plausible, indicating that the manager has to borrow money to invest in risky assets even in the case of over-funding. Of course, this can only be due to a high value of the instantaneous volatility of $P$ with respect to that of the stock, and to the existence of a positive correlation.

We may wonder why the manager is going to invest in risky assets if he or she can attain the same objectives of minimizing risks by investing in fixed rent and if the region of stability in the former case is smaller than in the latter, as shown in Remarks 3.2 and 4.2. The reasons for doing so are contained in the two following results, where we suppose that Hypothesis B holds in the fixed rent case and that Hypothesis B' holds in the other case.

Corollary 4.1. If Hypothesis A holds, then the rate of convergence of the expected value of the fund to the expected value of actuarial liability in the long run is greater when the manager invests both in the bond and risky assets than when he or she invests only in fixed rent.

Hence, on average, the stabilization of the plan is faster with the selection of an adequate portfolio involving risky assets and a constant bond, in contrast to the case of investing only at a fixed and deterministic rate of interest.

Another interesting question is to compare the total averaged contribution rate made by the sponsoring employees through time, under the two types of management. 
Corollary 4.2. If Hypothesis $\mathrm{A}$, (9) and (21) hold, then, when $F_{0}<\mathrm{AL}_{0}$ the total expected value of the optimal contribution rate is less when the manager invests in both the bond and risky assets than when he or she invests only in fixed rent.

\section{Optimal pension funding with stochastic rate of return}

In this section we investigate what the optimal behavior of the pension manager is when there is no risk-free asset. As noted by Cairns (2000), some pension funds only use cash for short-term liquidity and not as an asset to invest in the long run. In general, funds use government bonds with fixed interest as low risk assets, but they are not risk-free. In this situation, we can ask ourselves what the technical rate of actualization is that is chosen by the actuaries, leading to a spread method of funding.

To simplify the model, let us consider that the whole wealth of the fund is invested in a single risky asset. This case cannot be handled from results established in Section 4, but the model is as specified in that section, without the bond $S^{0}$ and with $n=1$. Therefore, we now suppose that there is only one risky asset whose price is a geometric Brownian motion

$$
\mathrm{d} S(t)=b S(t) \mathrm{d} t+\sigma S(t) \mathrm{d} W_{1}(t),
$$

where $b>0$ and $\sigma>0$. The symbol $W_{1}$ denotes a standard Brownian motion. Remember, however, that $B$ is the standard Brownian motion intervening in the evolution of the benefits. We suppose that $W_{1}$ and $B$ are correlated, as in Section 4. That is to say, $\mathbb{E}\left(B(t) W_{1}(s)\right)=q \min (t, s)$, with $-1 \leq q \leq 1$. Hence we can write $B=\sqrt{1-q^{2}} W_{0}+q W_{1}$, where $\left(W_{0}, W_{1}\right)$ is a standard Brownian motion defined on the corresponding probability space.

The behavior of the wealth of the fund is given by the stochastic differential equation

$$
\mathrm{d} F(t)=F(t) \frac{\mathrm{d} S(t)}{S(t)}+(C(t)-P(t)) \mathrm{d} t,
$$

or

$$
\mathrm{d} F(t)=(b F(t)+C(t)-P(t)) \mathrm{d} t+\sigma F(t) \mathrm{d} W_{1}(t), \quad F(0)=F_{0} \geq 0 .
$$

As we already know, Hypothesis A allows us to eliminate $P$ and $\mathrm{NC}$ from the formulation

$$
\mathrm{d} F(t)=(b F(t)+\mathrm{SC}(t)+(\mu-\delta) \operatorname{AL}(t)) \mathrm{d} t+\sigma F(t) \mathrm{d} W_{1}(t) .
$$

The payoff is again given by the quadratic expression (5). Note that the fund is completely invested in the risky asset, so the only control variable is the supplementary cost.

The value function of the stochastic problem now becomes

$$
\hat{V}(F, \mathrm{AL})=\min _{\mathrm{SC} \in \mathcal{A}_{F, \mathrm{AL}}}\{J((F, \mathrm{AL}) ; \mathrm{SC}): \text { s.t. }(1),(22)\},
$$

where the class of admissible controls, $\mathcal{A}_{F, \mathrm{AL}}$, is defined as in the previous sections.

Since our main objective is to show that an adequate selection of $\delta$ leads to a spread method of funding, the following hypothesis is imposed.

Hypothesis $\mathbf{B}^{\prime \prime}$. The technical rate of valuation satisfies $\delta=b+\sigma^{2}-\eta q \sigma>0$.

Theorem 5.1. If Hypotheses $\mathrm{A}$ and $\mathrm{B}^{\prime}$ hold and if the inequality $2 \mu+\eta^{2}<\rho$ is satisfied, then the optimal rate of contribution is given by

$$
C^{*}(F, \mathrm{AL})=\mathrm{NC}+\frac{\gamma_{F F}}{\kappa} \mathrm{UAL},
$$


where $\gamma_{F F}$ is the unique positive solution to the equation

$$
\gamma_{F F}^{2}+\kappa\left(\rho-2 b-\sigma^{2}\right) \gamma_{F F}-\kappa(1-\kappa)=0 .
$$

Remark 5.1. As in the above sections, it is possible to obtain

$$
\mathbb{E}_{F_{0}, \mathrm{AL}_{0}} F(t)-a_{3} \mathbb{E}_{F_{0}, \mathrm{AL}_{0}} \mathrm{AL}(t)=\left(F_{0}-a_{3} \mathrm{AL}_{0}\right) \mathrm{e}^{\left(b-\gamma_{F F} / \kappa\right) t}
$$

with $a_{3}=\left(\gamma_{F F}+\kappa(\mu-\delta)\right) /\left(\gamma_{F F}+\kappa(\mu-b)\right)$, and therefore $\gamma_{F F}>\kappa b$ implies

$$
\lim _{t \rightarrow \infty}\left(\mathbb{E}_{F_{0}, \mathrm{AL}_{0}} F(t)-a_{3} \mathbb{E}_{F_{0}, \mathrm{AL}_{0}} \mathrm{AL}(t)\right)=0 .
$$

With a zero risk-free rate of interest, the expected value of the optimal fund minus the actuarial liability converges, in the long run to zero if, and only if, the constant $a_{3}=1$, that is to say, $\sigma=\eta q$. This is an important difference with respect to the case where there is a constant rate of return, where the expected value of the fund in the long run exactly matches the expected value of the actuarial liability. The fact that in general it is not possible to attain the expected value of the liabilities could be a consequence of Hypothesis B" and the use of a spread method of funding. Some other selection of $\delta$-maybe a non-constant one-could lead to an exact stabilization at the expense of a more complicated method of funding.

\section{Conclusions}

We have studied the problem of pension funding in a defined benefit pension plan from the perspective of a manager who wishes to keep the fund to prescribed targets with the aim of minimizing the solvency risk and the contribution rate risk. The liabilities outgo is subject to disturbances influencing the evolution of the pension management. We study three scenarios and determine the optimal behavior of the controller for each one. We find that it is always possible to choose - in a rather natural way - the technical rate of actualization such that the supplementary cost is proportional to the unfunded actuarial liability.

The paper shows that the expected value of the fund converges faster in the long run to the expected value of the actuarial liability when the agent diversifies her or his investment. The investment in high return, high risk assets decreases linearly as the fund increase towards the ideal value, but even when $F$ exactly matches AL, the optimal investment in risky assets is not null. This behavior implies taking more and more risks when the level of the fund is low, but it demands selling the risky assets short when the fund is operating with a large surplus.

An interesting question is to consider that the rate of return of the bond is not constant, but that it follows some stochastic process, as proposed by Vasicek (1977) or Cox et al. (1985). This is the approach taken in the recent papers of Boulier et al. (2001) and Deelstra et al. (2003) in defined contribution pension funds.

\section{Acknowledgements}

Financial support from Consejería de Educación y Cultura de la Junta de Castilla y León under project VA108/01 and Ministerio de Ciencia y Tecnología and FEDER under project BFM2002-00425 is gratefully acknowledged.

\section{Appendix A}

Proof of Proposition 2.1. If Hypothesis A holds, then the benefits are given by

$$
P(t)=P_{0} \mathrm{e}^{\left(\mu-\eta^{2} / 2\right) t+\eta B(t)}, \quad t \geq 0 .
$$


Therefore, for every $x \in[a, d]$ and $t \geq 0$ the following holds:

$$
\begin{aligned}
\mathbb{E}\left(P(t+d-x) \mid \mathcal{F}_{t}\right) & =P_{0} \mathbb{E}\left(\mathrm{e}^{\left(\mu-\eta^{2} / 2\right)(t+d-x)+\eta B(t+d-x)} \mid \mathcal{F}_{t}\right)=P_{0} \mathrm{e}^{\mu(t+d-x)} \mathbb{E}\left(\mathrm{e}^{-\eta^{2} / 2(t+d-x)+\eta B(t+d-x)} \mid \mathcal{F}_{t}\right) \\
& =P_{0} \mathrm{e}^{\mu(t+d-x)} \mathrm{e}^{-\left(\eta^{2} / 2\right) t+\eta B(t)}=P_{0} \mathrm{e}^{\mu(d-x)} \mathrm{e}^{\left(\mu-\eta^{2} / 2\right) t+\eta B(t)}=\mathrm{e}^{\mu(d-x)} P(t) .
\end{aligned}
$$

The first equality holds because $P_{0}$ is $\mathcal{F}_{0}$-measurable and the third because $\mathrm{e}^{-\left(\eta^{2} / 2\right) s+\eta B(s)}$ is an $\mathcal{F}_{t}$-martingale for $s \geq t$.

Therefore, defining

$$
\psi_{\mathrm{AL}}=\int_{a}^{d} \mathrm{e}^{(\mu-\delta)(d-x)} M(x) \mathrm{d} s, \quad \psi_{\mathrm{NC}}=\int_{a}^{d} e^{(\mu-\delta)(d-x)} m(x) \mathrm{d} s,
$$

the statement of the proposition follows. Finally, the relationship between $\psi_{\mathrm{AL}}$ and $\psi_{\mathrm{NC}}$ is obtained integrating by parts in the second integral and taking into account the properties of $M$ and that $M^{\prime}=m$.

Proof of Theorem 3.1. For the problem of optimal pension funding established in Section 3, the HJB equation becomes:

$$
\rho V=\min _{\mathrm{SC}}\left\{(r F+\mathrm{SC}+(\mu-\delta) \mathrm{AL}) V_{F}+\mu \mathrm{AL} V_{\mathrm{AL}}+\frac{1}{2}\left(\eta^{2}\right) \mathrm{AL}^{2} V_{\mathrm{AL}, \mathrm{AL}}+\kappa \mathrm{SC}^{2}+(1-\kappa)(\mathrm{AL}-F)^{2}\right\} .
$$

If there is a smooth solution $V$ of this equation, strictly convex with respect to $(F, \mathrm{AL})$, then the minimizer value of the supplementary cost rate is given by

$$
\widehat{\mathrm{SC}}\left(V_{F}\right)=-\frac{V_{F}}{2 \kappa}
$$

that is, $\hat{C}\left(V_{F}\right)=\mathrm{NC}-V_{F} / 2 \kappa$. We first substitute this value in (A.1) and then we try a solution of the form

$$
V(F, \mathrm{AL})=\alpha_{F F} F^{2}+\alpha_{\mathrm{AL}, \mathrm{AL}} \mathrm{AL}^{2}+\alpha_{F, \mathrm{AL}} F \mathrm{AL} .
$$

The following three equations are obtained for the above coefficients

$$
\begin{aligned}
& \alpha_{F F}^{2}+\kappa(\rho-2 r) \alpha_{F F}-\kappa(1-\kappa)=0, \quad-\alpha_{F, \mathrm{AL}} \alpha_{F F}+\kappa(\mu+r-\rho) \alpha_{F, \mathrm{AL}}+2 \kappa(\mu-\delta) \alpha_{F F}-2 \kappa(1-\kappa)=0, \\
& 4 \kappa\left(\rho-2 \mu-\eta^{2}\right) \alpha_{\mathrm{AL}, \mathrm{AL}}+\alpha_{F, \mathrm{AL}}^{2}-4 \kappa(\mu-\delta) \alpha_{F, \mathrm{AL}}-4 \kappa(1-\kappa)=0 .
\end{aligned}
$$

The first equation is precisely (8), which admits a positive solution. Hypothesis B implies the equality $-2 \alpha_{F F}=$ $\alpha_{F, \mathrm{AL}}$ and then, the second equation coincides exactly with the first one. Substituting these expressions in the third equation and subtracting this from the first one, $\alpha_{\mathrm{AL}, \mathrm{AL}}=\left((\rho-2 \mu) /\left(\rho-2 \mu-\eta^{2}\right)\right) \alpha_{F F} \geq \alpha_{F F}$, assuring that $V$ is non-negative.

According to Fleming and Soner (1993), to prove that the solution we have found of Eq. (A.1) is in fact the value function of the problem, it is sufficient to check that the transversality condition

$$
\lim _{t \rightarrow \infty} \mathrm{e}^{-\rho t} \mathbb{E}_{F_{0}, \mathrm{AL}_{0}} V\left(F^{*}(t), \mathrm{AL}(t)\right)=0
$$

holds, where $F^{*}$ is the optimal fund wealth

$$
\mathrm{d} F^{*}(t)=\left(\left(r-\frac{\alpha_{F F}}{\kappa}\right) F^{*}(t)+\left(\frac{\alpha_{F F}}{\kappa}+\mu-r\right) \operatorname{AL}(t)\right) \mathrm{d} t
$$

obtained after substitution in (4) of the expression for $\mathrm{SC}^{*}$ given in (18). Since $V$ is a homogeneous quadratic polynomial in $F$ and $\mathrm{AL}$, it is necessary to calculate the expected values of $\left(F^{*}\right)^{2}, F^{*} \mathrm{AL}$ and $\mathrm{AL}^{2}$ conditioned 


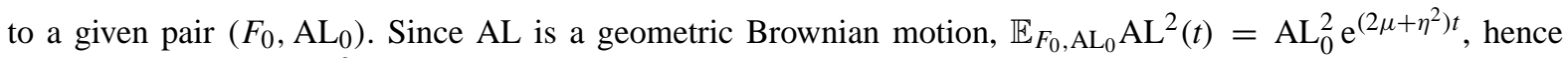
$\lim _{t \rightarrow \infty} \mathrm{e}^{-\rho t} \mathbb{E}_{F_{0}, \mathrm{AL}_{0}} \mathrm{AL}^{2}(t)=0$ if and only if (6) holds.

Following Arnold (1974, p. 140), the functions defined by $g(t)=\mathbb{E}_{F_{0}, \mathrm{AL}_{0}}\left(F^{*} \mathrm{AL}\right)(t)$ and $h(t)=\mathbb{E}_{F_{0}, \mathrm{AL}_{0}}\left(F^{*}\right)^{2}(t)$ satisfy the linear differential equations

$$
g^{\prime}(t)=\left(r-\frac{\alpha_{F F}}{\kappa}+\mu\right) g(t)+\left(-r+\frac{\alpha_{F F}}{\kappa}+\mu\right) \varphi(t), \quad h^{\prime}(t)=2\left(r-\frac{\alpha_{F F}}{\kappa}\right) h(t)+2\left(\frac{\alpha_{F F}}{\kappa}+\mu-r\right) g(t)
$$

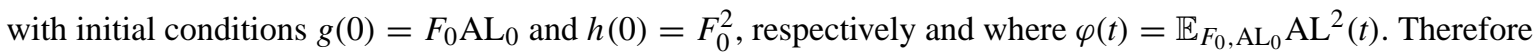

$$
g(t)=\left(F_{0}-a_{1} \mathrm{AL}_{0}\right) \mathrm{AL}_{0} \mathrm{e}^{\left(r-\alpha_{F F} / \kappa+\mu\right) t}+a_{1} \varphi(t)
$$

with $a_{1}=\left(\alpha_{F F}+\kappa(\mu-r)\right) /\left(\alpha_{F F}+\kappa\left(\mu-r+\eta^{2}\right)\right)$. Assuming inequality (6) we have $\lim _{t \rightarrow \infty} \mathrm{e}^{-\rho t} \mathbb{E}_{F_{0}, \mathrm{AL}_{0}}\left(F^{*} \mathrm{AL}\right)(t)$ $=0$ if and only if $r-\alpha_{F F} / \kappa+\mu<\rho$ hold. To prove this, note that the definition of $\alpha_{F F}$ as the unique positive solution of (8) implies $r-\alpha_{F F} / \kappa<\rho / 2$. Taking into account (6) we obtain $r-\alpha_{F F} / \kappa+\mu \leq r-\alpha_{F F} / \kappa+\mu+\eta^{2} / 2<\rho$. On the other hand

$$
h(t)=\left(F_{0}^{2}-2 F_{0} \mathrm{AL}_{0}+\left(2 a_{1}-a_{1} a_{2}\right) \mathrm{AL}_{0}^{2}\right) \mathrm{e}^{2\left(r-\alpha_{F F} / \kappa\right) t}+2 g(t)-\left(2 a_{1}-a_{1} a_{2}\right) \varphi(t)
$$

with $a_{2}=\left(\alpha_{F F}+\kappa(\mu-r)\right) /\left(\alpha_{F F}+\kappa\left(\mu-r+\eta^{2} / 2\right)\right)$. Hence $\lim _{t \rightarrow \infty} \mathrm{e}^{-\rho t} h(t)=0$ if and only if (6) and $2 r-2 \alpha_{F F} / \kappa<\rho$ hold. Again the latter inequality is obtained from (8) and (6). This ends the proof.

Proof of Theorem 4.1. The HJB equation is now

$$
\begin{aligned}
\rho V= & \min _{\mathrm{SC}, \bar{\lambda}}\left\{\left(r F+\bar{\lambda}^{\top}(\bar{b}-r \overline{1})+\mathrm{SC}+(\mu-\delta) \mathrm{AL}\right) V_{F}+\mu \mathrm{AL} V_{\mathrm{AL}}+\frac{1}{2} \bar{\lambda}^{\top} \Sigma \bar{\lambda} V_{F F}+\frac{1}{2} \eta^{2} \mathrm{AL}^{2} V_{\mathrm{AL}, \mathrm{AL}}\right. \\
& \left.+\eta \mathrm{AL} \bar{\lambda}^{\top} \sigma \bar{q} V_{F, \mathrm{AL}}+\kappa \mathrm{SC}^{2}+(1-\kappa)(F-\mathrm{AL})^{2}\right\} .
\end{aligned}
$$

If there is a smooth solution $V$ of the Eq. (A.3), strictly convex, then the maximizers values of the contribution rate and the investment rates are given by

$$
\widehat{\mathrm{SC}}\left(V_{F}\right)=-\frac{V_{F}}{2 \kappa}, \quad \hat{\bar{\lambda}}\left(V_{F}, V_{F F}, V_{\mathrm{AL}, \mathrm{AL}}\right)=-\Sigma^{-1}(\bar{b}-r \overline{1}) \frac{V_{F}}{V_{F F}}-\eta \mathrm{AL} \sigma^{-\top} \bar{q} \frac{V_{F, \mathrm{AL}}}{V_{F F}},
$$

respectively. The structure of the equation obtained once we have substituted this values for SC and $\bar{\lambda}$ in (A.2), suggests a quadratic homogeneous solution

$$
V(F, \mathrm{AL})=\beta_{F F} F^{2}+\beta_{\mathrm{AL}, \mathrm{AL}} \mathrm{AL}^{2}+\beta_{F, \mathrm{AL}} F \mathrm{AL} .
$$

Imposing this solution the following set of three equations is obtained for the coefficients

$$
\begin{aligned}
& \beta_{F F}^{2}+\kappa\left(\rho+\bar{\theta}^{\top} \bar{\theta}-2 r\right) \beta_{F F}-\kappa(1-\kappa)=0, \\
& -\beta_{F, \mathrm{AL}} \beta_{F F}+\kappa\left(\mu+r-\rho-\bar{\theta}^{\top} \bar{\theta}-\eta \bar{q}^{\top} \bar{\theta}\right) \beta_{F, \mathrm{AL}}+2 \kappa(\mu-\delta) \beta_{F F}-2 \kappa(1-\kappa)=0, \\
& 4 \kappa\left(\rho-2 \mu-\eta^{2}\right) \beta_{\mathrm{AL}, \mathrm{AL}} \beta_{F F}+\beta_{F F} \beta_{F, \mathrm{AL}}^{2}-4 \kappa(\mu-\delta) \beta_{F, \mathrm{AL}} \beta_{F F}+\kappa\left(\eta^{2} \bar{q}^{\top} \bar{q}+2 \eta \bar{q}^{\top} \bar{\theta}+\bar{\theta}^{\top} \bar{\theta}\right) \beta_{F, \mathrm{AL}}^{2} \\
& \quad-4 \kappa(1-\kappa) \beta_{F F}=0 .
\end{aligned}
$$

Although it would be possible to solve the above equations with full generality, we wish to determine values of the parameters of the problem leading to a spread method of funding. A view to the optimal contribution rate helps us to find how to choose the technical rate of actualization $\delta$. From (A.4)

$$
\mathrm{SC}=-\frac{1}{2 \kappa}\left(2 \beta_{F F} F+\beta_{F, \mathrm{AL}} \mathrm{AL}\right),
$$


hence the supplementary cost is proportional to the unfunded actuarial liability, if and only if, $\beta_{F, \mathrm{AL}}=-2 \beta_{F F}$. But then, the first and second equations can have a solution, if and only if, $\delta=r+\eta \bar{q}^{\top} \bar{\theta}$. Substituting these expressions in the third equation and subtracting from the first one

$$
\beta_{\mathrm{AL}, \mathrm{AL}}=\frac{\rho-2 \mu-\eta^{2} \bar{q}^{\top} \bar{q}}{\rho-2 \mu-\eta^{2}} \beta_{F F} \geq \beta_{F F},
$$

assuring the non-negativity of $V$.

As in the proof of Theorem 3.1, we proceed by showing that the transversality condition holds. The reasonings are very similar, the only change being the dynamics of the optimal fund, $F^{*}$

$$
\begin{aligned}
\mathrm{d} F^{*}(t)= & \left(\left(r-\bar{\theta}^{\top} \bar{\theta}-\frac{\beta_{F F}}{\kappa}\right) F^{*}(t)+\left(-r+\bar{\theta}^{\top} \bar{\theta}+\frac{\beta_{F F}}{\kappa}+\mu\right) \operatorname{AL}(t)\right) \mathrm{d} t \\
& +\left(-\bar{\theta}^{\top} F^{*}(t)+\left(\bar{\theta}^{\top}+\eta \bar{q}^{\top}\right) \operatorname{AL}(t)\right) \mathrm{d} W(t) .
\end{aligned}
$$

It is easy to show that

$$
\mathbb{E}_{F_{0}, \mathrm{AL}_{0}}\left(F^{*} \mathrm{AL}\right)(t)=\left(F_{0}-a_{1} \mathrm{AL}_{0}\right) \mathrm{AL}_{0} \mathrm{e}^{\left(r-\bar{\theta}^{\top} \bar{\theta}-\beta_{F F} / \kappa+\mu-\eta \bar{q}^{\top} \bar{\theta}\right) t}+a_{1} \varphi(t),
$$

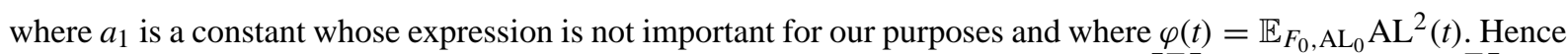
$\lim _{t \rightarrow \infty} \mathrm{e}^{-\rho t} \mathbb{E}_{F_{0}, \mathrm{AL}_{0}}\left(F^{*} \mathrm{AL}\right)(t)=0$, if and only if, both (17) and the inequality $r-\bar{\theta}^{\top} \bar{\theta}-\beta_{F F} / \kappa+\mu-\eta \bar{q}^{\top} \bar{\theta}<\rho$ simultaneously hold. The latter condition follows from (17) and, by the definition of $\beta_{F F}$, as the positive solution of (20). To see this, in the first place we observe that

$$
-\eta \bar{q}^{\top} \bar{\theta} \leq \eta \overline{1}^{\top} \bar{\theta}=\frac{1}{2}\left(\bar{\theta}^{\top} \bar{\theta}+\eta^{2}-(\bar{\theta}-\eta \overline{1})^{\top}(\bar{\theta}-\eta \overline{1})\right) \leq \frac{1}{2} \bar{\theta}^{\top} \bar{\theta}+\frac{1}{2} \eta^{2}
$$

because $-1 \leq q_{i} \leq 1$. Now (20) implies $r-\bar{\theta}^{\top} \bar{\theta} / 2-\beta_{F F} / \kappa<\rho / 2$. These inequalities and (17) justify the claim. On the other hand

$$
\begin{aligned}
\mathbb{E}_{F_{0}, \mathrm{AL}_{0}}\left(F^{*}\right)^{2}(t)= & \left(F_{0}^{2}-2 F_{0} \mathrm{AL}_{0}+\left(2 a_{1}-a_{1} a_{2}-a_{3}\right) \mathrm{AL}_{0}^{2}\right) \mathrm{e}^{2\left(r-\left(\beta_{F F} / \kappa\right)-\left(\bar{\theta}^{\top} \bar{\theta} / 2\right)\right) t} \\
& +2 \mathbb{E}_{F_{0}, \mathrm{AL}_{0}}\left(F^{*} \mathrm{AL}\right)(t)-\left(2 a_{1}-a_{1} a_{2}-a_{3}\right) \varphi(t),
\end{aligned}
$$

where $a_{2}$ and $a_{3}$ are constants. The arguments leading to $\lim _{t \rightarrow \infty} \mathrm{e}^{-\rho t} \mathbb{E}_{F_{0}, \mathrm{AL}_{0}}\left(F^{*}\right)^{2}(t)=0$ under the hypotheses of the theorem are quite similar to that shown above.

Proof of Corollary 4.1. With the explicit expressions of $\alpha_{F F}$ and $\beta_{F F}$, it is easy to verify that the rate of convergence in the first case, $\left|r-\bar{\theta}^{\top} \theta-\left(\beta_{F F} / \kappa\right)\right|$, is greater than in the second, which is given by $\left|r-\left(\alpha_{F F} / \kappa\right)\right|$.

Proof of Corollary 4.2. Let us call case 1 the situation where there is no diversification in the investment and case 2 the contrary. Let $C_{i}$ be the total expected value of the contribution rate in case $i, i=1,2$. Taking into account the expressions for the expected value of the unfunded actuarial liability given in Remarks 3.2 and 4.2, we have

$$
\begin{aligned}
C_{1}-C_{2} & =\frac{\mathrm{UAL}_{0}}{\kappa} \int_{0}^{\infty}\left(\alpha_{F F} \mathrm{e}^{\left(r-\alpha_{F F} / \kappa\right) s}-\beta_{F F} \mathrm{e}^{\left(r-\beta_{F F} / \kappa-\bar{\theta}^{\top} \bar{\theta}\right) s}\right) \mathrm{d} s \\
& =\frac{\kappa \mathrm{UAL}_{0}}{\left(\kappa r-\alpha_{F F}\right)\left(\kappa\left(r-\bar{\theta}^{\top} \bar{\theta}\right)-\beta_{F F}\right)}\left(r\left(\beta_{F F}-\alpha_{F F}\right)+\alpha_{F F} \bar{\theta}^{\top} \bar{\theta}\right),
\end{aligned}
$$

which is positive by the assumptions made on the parameters.

Proof of Theorem 5.1. The proof is quite similar to that of Theorems 3.1 and 4.1, so it is omitted. 


\section{References}

Arnold, L., 1974. Stochastic Differential Equations: Theory and Applications. Wiley, New York.

Boulier, J.F., Huang, S., Taillard, G., 2001. Optimal management under stochastic interest rates: the case of a protected defined contribution pension fund. Insurance: Mathematics and Economics 28, 173-189.

Boulier, J.F., Michel, S., Wisnia, V., 1996. Optimizing investment and contribution policies of a defined benefits pension fund. In: Proceedings of the Sixth AFIR International Colloquium, vol. 1, pp. 593-607.

Boulier, J.F., Trussant, E., Florens, D., 1995. A dynamic model for pension funds management. In: Proceedings of the Fifth AFIR International Colloquium, vol. 1, pp. 361-384.

Bowers, N.L., Gerber, H.U., Hickman, J.C., Jones, D.A., Nesbitt, C.J., 1986. Actuarial Mathematics. The Society of Actuaries, Itaca.

Cairns, A.J.G., 1995. Pension funding in a stochastic environment: the role of objectives in selecting an asset-allocation strategy. In: Proceedings of the Fifth AFIR International Colloquium, vol. 1, pp. 429-453.

Cairns, A.J.G., 1996. Continuous-time stochastic pension fund modelling. In: Proceedings of the Sixth AFIR International Colloquium, vol. 1, pp. 609-624.

Cairns, A.J.G., 2000. Some notes on the dynamics and optimal control of stochastic pension fund models in continuous time. Astin Bulletin 30, $19-55$.

Chang, S.C., Tzeng, L.T., Miao, J.C.Y., 2003. Pension funds incorporating downside risks. Insurance: Mathematics and Economics 32, $217-228$.

Constantinides, G., 1978. Market risk adjustment and project valuation. Journal of Finance 33, 603-616.

Cox, J.C., Ingersoll, J.E., Ross, S.A., 1985. A theory of the term structure of interest rates. Econometrica 53, 385-408.

Deelstra, G, Grasselli, M., Koehl, P.F., 2003. Optimal investment strategies in the presence of a minimum guarantee. Insurance: Mathematics and Economics 33, 189-207.

Fleming, W.H., Soner, H.M., 1993. Controlled Markov Processes and Viscosity Solutions. Springer-Verlag, New York.

Haberman, S., 1993. Stochastic investment returns and the present value of future contributions in defined benefits pension schemes. Actuarial Research Paper 49, City University.

Haberman, S., 1997. Stochastic investment returns and contribution rate risk in a defined benefits pension scheme. Insurance: Mathematics and Economics 19, 127-139.

Haberman, S., Sung, J.H., 1994. Dynamics approaches to pension funding. Insurance: Mathematics and Economics 15, 151-162.

Josa-Fombellida, R., Rincón-Zapatero, J.P., 2001. Minimization of risks in pension funding by means of contribution and portfolio selection. Insurance: Mathematics and Economics 29, 35-45.

Merton, R.C., 1971. Optimal consumption and portfolio rules in a continuous-time model. Journal of Economic Theory 3, $373-413$.

O'Brien, T.V., 1987. A two parameter family of pension contribution functions and stochastic optimization. Insurance: Mathematics and Economics 6, 129-134.

Øksendal, B., 1998. Stochastic Differential Equations, 5th ed. Springer-Verlag, New York.

Owadally, M.I., Haberman, S., 1999. Pension fund dynamics and gains/losses due to random rates of investment return. North American Actuarial Journal 3, 105-117.

Shimko, D.C., 1992. Finance in Continuous Time: A Primer. Kolb Publishing Company, Miami.

Taylor, G., 2002. Stochastic control of funding systems. Insurance: Mathematics and Economics 30, 323-350.

Vasicek, O.A., 1977. An equilibrium characterization of the term structure. Journal of Financial Economics 5, 177-188.

Vigna, E., Haberman, S., 2001. Optimal investment strategy for defined contribution pension schemes. Insurance: Mathematics and Economics $28,253-262$. 